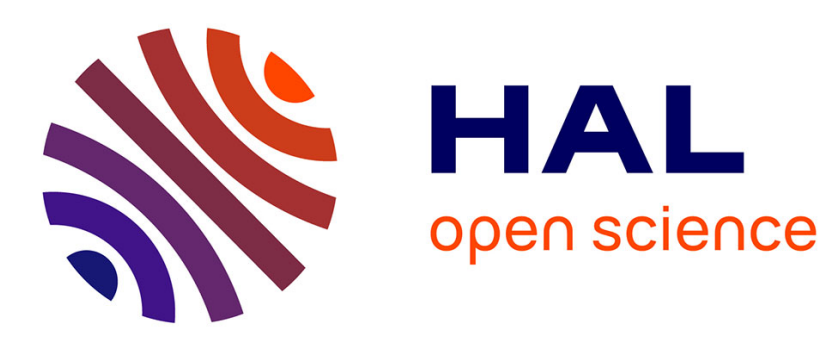

\title{
Influence of Sparkle and Saccades on Tongue Electro-Stimulation-Based Vision Substitution of 2D Vectors
}

\author{
Abdessalem Chekhchoukh, Nicolas Glade
}

\section{- To cite this version:}

Abdessalem Chekhchoukh, Nicolas Glade. Influence of Sparkle and Saccades on Tongue ElectroStimulation-Based Vision Substitution of 2D Vectors. Acta Biotheoretica, 2012, 60 (1), pp.41. 10.1007/s10441-012-9148-2 . hal-00724897

\section{HAL Id: hal-00724897 \\ https://hal.science/hal-00724897}

Submitted on 23 Aug 2012

HAL is a multi-disciplinary open access archive for the deposit and dissemination of scientific research documents, whether they are published or not. The documents may come from teaching and research institutions in France or abroad, or from public or private research centers.
L'archive ouverte pluridisciplinaire HAL, est destinée au dépôt et à la diffusion de documents scientifiques de niveau recherche, publiés ou non, émanant des établissements d'enseignement et de recherche français ou étrangers, des laboratoires publics ou privés. 


\title{
Influence of sparkle and saccades on tongue electro-stimulation-based vision substitution of $2 \mathrm{D}$ vectors
}

\author{
Abdessalem Chekhchoukh • Nicolas \\ Glade
}

Received : date / Accepted : date

\begin{abstract}
Vision substitution by electro-stimulation has been studied since the 60's beginning with P. Bach-y-Rita. Camera pictures or movies encoded in gray levels are displayed using an electro-stimulation display device on the surface of a body part, such as the skin or the tongue. Medical-technical devices have been developed on this principle to compensate for sensory-motor disabilities such as blindness or loss of balance, or to guide specific actions, such as surgery. However, the electrical signals of stationary or moving slowly moving objects, displayed on a Tongue Display Unit (TDU), are quickly lost due to saturation of receptors undergoing electostimulation. We propose to add random saccades or sparkle to the displayed visual scene to increase the quality of pattern recognition by the subjects. In the present experimental trimodal study (normal vision, TDU vision substitution, or both), we show that the presence of a moderate sparkle level enhances the perception of the direction of lines drawn on a TDU and reduces the response time.
\end{abstract}

Keywords Sensory substitution · Tongue Display Unit · Vision · Eye micro-saccades · Sparkle

This work was supported by the CSVSB (Pole of Chemistry, Biology, Health and BioEngineering) of the University of Grenoble.

\footnotetext{
A. Chekhchoukh · N. Glade

AGIM Laboratory

Laboratory of AGeing Imaging and Modeling

University of Grenoble - CNRS FRE 3405

Domaine de la Merci, 38700 La Tronche, France

Tel. : +33-4-56-52-00-27

Fax : +33-4-76-76-88-44

E-mail: Nicolas.Glade@ujf-grenoble.fr
} 


\section{Introduction}

The eyes are not limited to an arrangement of photoreceptors like electronic sensors of digital cameras. These biologicals sensor also come with a neuromuscular motion control. In addition to playing a stabilizing role of the eye and gaze control, this also allows a feature of modern eyes to function: a reflex called micro-saccades. The ocular micro-saccades were introduced after millions of years by evolution certainly at the onset of evolved predators (Rodieck 2003). Indeed, they allow the predator to detect and hunt immobile preys while former predators (like reptiles) can not. When an eye is maintained stationary and forced to fix a visual scene, as it is the case in the Troxler's fading effect (Troxler 1804), the scene perceived by the peripheral vision rapidly shades away and disappears. Eyes' photoreceptors are saturated when subjects to an unvarying stimulation. In these conditions, the signal blends with the surrounding perceived scene. This constitutes a problem in the development of vision substitution systems based on electrostimulation on the skin or the tongue. Eyes' moves, and in particular micro-saccades, avoid photoreceptors to be always stimulated by the same signal, so the scene is always refreshed and continues to be perceived. What is true for the eyes is certainly also true for other kinds of biological sensors.

Sensory and in particular vision substitution paradigm was introduced by Paul Bach-y-Rita in the 60's (Bach-y-Rita et al 1969). This paradigm supposes that, when a biological sensory system is defective (e.g. vision), the information to which it is related can be (i) grabbed by an artificial sensor (resp. a digital camera), (ii) transformed in a different physical stimulation that can be exploited by a functional biological sensory system (resp. the tactile-kinesthetic system), and then (iii) handled by the central nervous system via the corresponding nervous paths (resp. somesthetic nerves). In practice, camera pictures or movies encoded in gray levels are displayed via an electro-stimulation device on the surface of a body part such as the skin or the tongue. Medico-technical devices are developed based on this principle using a TDU (the tongue display unit is composed of a control box and a square matrix of about $10 \times 10$ electrodes or taxels for tactile pixels) to take care of sensory-motor disabilities like blindness or macula degeneracy due to aging (Bach-y-Rita et al 1969, 1998; Sampaio et al 2001; Kupers and Ptito 2004; Ptito et al 2005; Danilov and Tyler 2005; Kercel 2005; Arnoldussen et al 2008), to allow equilibrium rehabilitation (Vuillerme et al 2007, 2008a; Vuillerme and Cuisinier 2008; Vuillerme et al 2008b), to prevent the formation of pressure sores (Vuillerme et al 2007; Chenu et al 2009; Chenu 2009),or even to guide specific actions like in surgery or other acts of trajectory tracking or of target localization requiring a guiding assistance (Robineau et al 2007; Robineau 2009; Bluteau 2010).

However, when conducting experiments in vision substitution (by electrostimulation) on the recognition of stationary and spatially localized information, we are quickly disappointed by the difficulty of maintaining the perception of the signal for more than a few seconds. Due to the saturation of the 
receptors, a signal maintained at one location is quickly lost. Our hypothesis is that mimicking the refresh function of the eyes by addition of a refreshing factor like saccades to the visual substitution signals sent to the TDU would help to perceive it. So, we propose here to add the equivalent of eye micro-saccades, i.e. local saccades or sparkle of the displayed signal so as to increase the quality of perception of vectorial signals displayed on the tongue with a TDU . Their efficiency is evaluated by measuring the sensitivity (response times and angle standard deviation) of healthy people in the direction of straight lines drawn either on a computer monitor (a VDU, for visual display unit) or on the TDU, or both (bimodality).

\section{Materials and methods}

\subsection{Graphics rendering}

\subsubsection{Graphics renderer}

Displaying quality graphics on a $12 \times 12$ display unit is not easy. Since we could not get good results with classical graphics rendering softwares like OpenGL and MESA GL, we develop and use a software graphics renderer called AVC (for Array Visu Context), more adapted to the low resolution of our device. In AVC, all algorithms implying colors were adapted to work in the HLS (Hue, Luminance, Saturation) mode which is well-adapted for representing voltage levels. Voltages seen as color components are floating values of luminance comprised between 0 and 1 (and saturation is set to 1).

\subsubsection{Micro-saccades and sparkle}

Saccades and sparkle are encoded as post-rasterization operations in the graphics renderer (Fig. 1). Saccades cause the entire rasterized scene to move randomly from a reference position to the positions in its neighborhood, the distance (a floating value comprised between 0 and $n$ taxel) and the frequency of the jumps following Gaussian probability laws. Jump directions are drawn according to a uniform probability. The sparkle effect is obtained similarly except that the random move of the scene is replaced by a random change in luminance of each of its points. A Gaussian probability law makes the luminance varying more or less intensely around its original value. Its frequency is also adjustable. In the present experiments we fixed the frequency of saccades or sparkle to the maximum (100\% of the time) and for each we chose to use 4 different intensities : 0\% (absence of factor), 30\%, 60\% and 100\% (maximum level of the factor) of the maximum standard deviation from the current (reference) position (maximum standard deviation of distance set to 1 taxel), or $0 \%$ (absence of factor), 30\%, 60\% and 100\% (maximum level of the factor) of the maximum standard deviation from the current luminance (maximum standard deviation of luminance set to 1.0 unit of luminance) in the limits of 
the values $[0.0,1.0])$. In the statistical analysis of our measurements, we were not able to distinguish the results obtained at $30 \%$ and $60 \%$ of saccades or sparkle, so we grouped them in a new class called 'moderate level'.

\subsection{Voltage calibration}

The sensitivity of the tongue to electric stimulations (Marlow et al 1965; Ringel and Ewanowski 1965; Lass et al 1972; Maeyama and Plattig 1989) is not the same for everyone. Moreover, this sensitivity varies along the surface of a tongue : coarsely the tongue is more sensitive at its apex compared to its depth part. As important as it is to help "see" and discriminate properly and comfortably stimulation, the calibration step is not an easy problem. Gradual adjustment by static stimulation, region by region, so that perception is uniform over the entire surface of the tongue, has been used so far (Vuillerme et al 2007, 2008a; Vuillerme and Cuisinier 2008; Vuillerme et al 2008b; Chenu et al 2009; Robineau et al 2007; Robineau 2009; Chenu 2009). However, we found that this protocol is not very effective for visual substitution. In particular, incorrect settings in the calibration matrix can be easily perceived locally in the case of scenes with moving objects. The dynamical method we used avoids the saturation of tongue receptors during the calibration process, allows a better evaluation of the intensities by the subject and makes him possible to compare easily the stimuli between different regions of the tongue.

A default matrix of voltages is used on the basis of an antero-posterior gradient, the lower $(1$ volt $)$ at the apex, the most intense $(10$ volts $)$ in the back of the tongue. The subject can display horizontal or vertical moving lines (constituted of points of equal intensity set to 1 ) or a point moving horizontally or vertically at a certain speed (adjustable). The signal must be perceived uniformly. So as to adjust readily, quickly and precisely the calibration matrix, the subject uses, in combination with a keyboard key for increasing or decreasing the voltage, either a graphic pen on a tablet or his finger on a touch-pad whose surfaces represent the TDU surface. The calibration matrices of a user are saved each time, before and after an experiment, and can be compared. We calculated an average calibration matrix from the set of matrices of the different subjects who participated in this experiment (Fig. 2 A, B, C and D). Their profile, an increasing anterior-posterior gradient, is comparable to the sensitivity map (Fig. 2 E) of the tongue reconstructed by Robineau (2009) from the literature data (Marlow et al 1965; Ringel and Ewanowski 1965; Lass et al 1972; Maeyama and Plattig 1989).

\subsection{Experimental protocol}

After a calibration step (see above), subjects were asked to reproduce the signal perceived, the faster they can, by a drawing on a graphic pen tablet (Wacom Graphire). 
Initially, the VDU (a 21" monitor) or the TDU are blank, i.e. no signal is sent to the devices. For each experimental measurement, the experimenter presses a button and the signal, a straight line of intensity 1 , is drawn on a display device. The line is displayed on the TDU using the totality of its surface $(12 \times 12$ electrodes or taxels $)$ or on a VDU window of $300 \times 300$ pixels $(8 \times 8$ $\mathrm{cm}$ ) where $12 \times 12$ squares (of $25 \times 25$ pixels each) represent the electrodes of the TDU (Fig. 1). Three modalities of scene restitution are considered : the VDU (visual mode), the TDU, or both of them at the same time (bimodal). The subject uses a pen to draw the answer (the direction of the line) on the graphic pen tablet (angle indications are shown on the drawing surface of the tablet). The drawing taken into account is a line defined by two points corresponding to the first contact of the pen on the tablet and the point when the contact is broken.

At least three sessions spaced by three days were planned for each subject, so as to measure all the subjects during this period and for the subjects to rest in between (at least one day between subject sessions). The angles of the lines drawn by the subjects and their response times were recorded, both constituting one measurement. During each session, after calibration, a series of 210 measurements is realized corresponding to the following condition : [3 modalities (VDU, TDU, bimodal) $] \times[10$ measurements without saccades and without sparkle, 30 measurements with saccades (30\%, 60\% and 100\%), 30 measurements with sparkle $(30 \%, 60 \%$ and 100\%)]. A total of 6930 measurements were yielded from 14 healthy and adult subjects, 7 males and 7 females, between 22 and 36 years old including biologists and computer scientists, i.e. persons who show different skill levels in the use of computer equipments (This parameter can partly explain the variability in the errors made in the drawings using the graphic pen tablet and the variability in response times). All angles are reduced to angles between $0^{\circ}$ and $90^{\circ}$ in order to correct a left-to-right mirror effect in the perception by some subjects of the direction of the line (this observation will be analyzed in another article). Finally, at the end of the 3 sessions, a series of questions were proposed to the subjects so as to evaluate their own feelings about the device, the experiment and their perception of the objects displayed on the TDU. In particular, we asked them which directions they perceived at most : vertical (along the antero-posterior axis of the tongue), horizontal or diagonal lines.

\section{Results}

Fig. 3 shows two important results. First, when the stimulus drawn lines get close to diagonals $\left(45^{\circ}, 135^{\circ}, 225^{\circ}\right.$ and $\left.315^{\circ}\right)$ the angle deviation grows (Fig. 3 Left). This indicates a preferential perception of the line patterns on the tongue. A quiz concerning the feeling of vertical, horizontal or diagonal directions by the subjects when the lines are displayed on the TDU clearly shows their preference to vertical lines instead of diagonal and horizontal ones (Fig. 3 Right). It is possible that the subjects find easier to draw vertical 
or horizontal lines compared to diagonal ones, perhaps due to a more difficult motion of their hand in the diagonal directions. In addition, vertical lines stimulate the apex of the tongue which is the most sensitive part of the tongue. The second noticeable point is that this phenomena is not only limited to the TDU modality but also occurs when the lines are drawn on both the TDU and the screen (bimodal perception). This means that, under the conditions of bimodal perception, the subjects are very sensitive to the displays on the TDU, although the VDU is a priori a modality that must be better perceived.

A distribution analysis of the angle standard deviations depending on the modality of perception and on the presence or absence of moderate levels of saccades and sparkle is given in Fig. 4 while a distribution of response times is given in Fig. 5. Of course the deviation is more important and the time to answer is greater (the cognitive loading is increased) when the subjects see the lines via the TDU instead of the screen. The tongue-based vision is not as precise as eye vision and the subjects need more time to identify the direction of the static lines, especially without any prior experience of the TDU. What was not expected is that the deviation also increases in the bimodal restitution of the drawing. As seen before in Fig. 3, the perception of the directions by the subjects, although facilitated by a direct vision on the screen, is visibly disturbed by bimodal vision. This is also confirmed by the analysis of response times which show a shift towards longer response times as it is the case for the TDU use.

The distributions of angle deviations in TDU mode show that saccades cause an increase of deviations while moderate sparkle seems to have at least no negative effect on the perception of directions. However, both saccades and sparkle reduce considerably the response times. An analysis of variance (two-way ANOVA with interactions, using the $R$ software and the library car) completed by a posteriori tests is summarized in Table. 1. The a posteriori tests used are unilateral pairwise Wilcoxon rank tests (angle standard deviations do not follow normal distributions) with Bonferroni p-value adjustment method. This study confirms the results on the effect of moderate levels of saccades and sparkle on the perception of directions. We analyzed the effect of 3 factors : 'visu' (VDU, TDU, Bimodal), 'saccades' or 'sparkle' (absence, moderate, strong), and their interactions noted 'sparkle:visu' and 'saccades:visu'.

The statistical analysis shows that the presence of saccades (moderate or strong) induces a decrease of the quality of perception (increase of angle deviation medians by 5 to $10^{\circ}$ on average) in bimodal vision and vision substitution. On the contrary, intermediate levels of sparkle (intensities of $30 \%$ and $60 \%$ taken together) seem to enhance the perception. Although the effect of sparkle on the average angle deviations is weak (about $1^{\circ}$ less on average), it is significant (ANOVA p-value of $9.610^{-3}$ confirmed by a a posteriori unilateral Wilcoxon rank test with a p-value of $4.710^{-3}$ ) and is particularly noticeable on important deviations (decrease by about $10^{\circ}$ in angle deviations up to $50^{\circ}$ ).

Moreover, as shown in Fig. 5, both sparkle and saccades when present at moderate or strong levels have an important and very significant effect on time response, in particular for saccades with a p-value close to 0 . In vision 
substitution (TDU), time response medians reduce on average from $2400 \mathrm{~ms}$ (median value ; TDU only) by $500 \mathrm{~ms}$ to $1900 \mathrm{~ms}$ (TDU + sparkle or saccades): decrease of median by 350 to $470 \mathrm{~ms}$ due to sparkle, and by 500 to $650 \mathrm{~ms}$ due to saccades, depending on their intensity. The effect also exists in bimodal vision with a average decrease of median by about $130 \mathrm{~ms}$ : median decreases by 70 to $110 \mathrm{~ms}$ due to sparkle, and by 130 to $200 \mathrm{~ms}$ due to saccades. There is no effect of these factors in normal vision (median of $1210 \mathrm{~ms}$ ).

\section{Conclusions}

We found a manner to increase the perception by - at least - healthy subjects of information displayed on electrostimulation matrices. The sparkling of visual scenes reduces the standard deviation on the perception of the direction of static lines. The optimal range is a moderate sparkle intensity comprised between $30 \%$ and $60 \%$. Although a role similar to that of sparkle, no beneficial effect of saccades on the quality of perception has been found. The relocation of the drawings around their original position is probably the cause of the loss of sensitivity of perception observed. We think that this is due to the low resolution of the matrix $(12 \times 12)$ : taxels are spaced by $2 \mathrm{~mm}$, meaning that the drawing of a line subjected to lateral saccades occupies a region of at least $4 \mathrm{~mm}$ large. A greater resolution (a matrix of $24 \times 24$ with taxels spaced by 0.5 $\mathrm{mm}$ is currently being fabricated) should solve this problem and the saccades could then have a similar effect to that of sparkle.

However saccades, as sparkle, were shown to reduce considerably the time necessary for the subjects to answer. This response time decreases by about $500 \mathrm{~ms}$ in vision substitution and by about $130 \mathrm{~ms}$ in bimodal vision. This indicates that the decision of the subjects is faster if not more accurate. There may be at least two reasons for this: the presence of these factors attracts the subjects, or the subjects feel better the signal because the receptors of the tongue are less saturated. This suggests a new experiment in which the effect of duration of exposure to electric signal before answer will be tested.

The sparkle effect is particularly useful in the restitution of stationary scenes since their corresponding electric signal on the TDU stimulates the same tongue receptors. Moving objects are indeed well perceived (Chekhchoukh et al 2011), the moving signal allowing the relaxation of stimulated receptors. This allows us to consider an modulated integration of this effect in the restitution of complex scenes consisting of moving and static objects : stationary objects will be displayed using sparkle, whereas those moving will not be subject to important sparkle levels anymore, the intensity of sparkle depending on the moving rate of the objects. This work combined with that on 2D and 3D object tracking (Chekhchoukh et al 2011) will help now to ameliorate the electrostimulation-based vision substitution and guidance devices. It furnishes an alternative to guiding methods and direct image camera outputs via a TDU. Vision substitution enhanced by sparkle is also a good manner to test and improve with a TDU the efficiency of prosthetic vision experiments 
(Tatur et al 2011). We indeed aim to propose a new approach of vision substitution based on 2D or 3D vectorial models of the environment (or of other kinds of information). Numerous applications are now envisaged, among them (i) a $3 \mathrm{D}$ vision device bringing into play the vectorization of digital camera acquired scenes or virtual pre-computed environments, (ii) a medical device of computer-aided surgery allowing to localize and track hidden targets in a body based on medical imagery as done in (J. Troccaz 2011) with a smart phone, or even (iii) in aero or astronautics to give spatial informations (e.g. axes and vectors, planes ...) or to help following moving targets like missiles or planes, or more simply (iV) video games for blind people.

Acknowledgements The authors would like to thank O. Chenu and Y. Payan (TIMCIMAG, CNRS UMR5525) for their assistance and stimulating discussions that were really helpful.

\section{References}

Arnoldussen A, Nemke C, Hogle R, Skinner K (2008) Brainport plasticity: balance and vision applications. In: Proceedings of the 9th International Conference on Low Vision

Bach-y-Rita P, Collins CC, Saunders FA, White B, Scadden L (1969) Vision substitution by tactile image projection. Nature 221:963-964

Bach-y-Rita P, Kaczmarek KA, Tyler ME, Garcia-Lara J (1998) Form perception with a 49-point electrotactile stimulus array on the tongue: a technical note. J Rehabil Res Dev 35:427-430

Bluteau J (2010) évaluation des effets de l'ajout d'interfaces haptiques sur le suivi manuel de trajectoires. PhD thesis, Université Joseph Fourier, Grenoble

Chekhchoukh A, Vuillerme N, Glade N (2011) Vision substitution and moving objects tracking in 2 and 3 dimensions via vectorial electro-stimulation of the tongue. In: Proceedings of the ASSISTH'11

Chenu O (2009) Conception et validation d'un dispositif de suppléance perceptive dédié à la prévention des escarres. $\mathrm{PhD}$ thesis, Université Joseph Fourier, Grenoble

Chenu O, Vuillerme N, Demongeot J, Payan Y (2009) A wireless lingual feedback device to reduce overpressures in seated posture: A feasibility study. Plos One 4:10:e7550

Danilov Y, Tyler M (2005) Brainport: An alternative input to the brain. J Integr Neurosci 4:537-550

J Troccaz MBIB G Le Bellego (2011) Using a smart phone for information rendering in computer-aided surgery. In: Springer (ed) Communications in Computer and Information Science, Proceedings of the HCI International 2011 
Kercel SW (2005) The wide-ranging impact of the work of paul bach-y-rita. J Integr Neurosci 4:403-406

Kupers R, Ptito M (2004) 'seeing' through the tongue: cross-modal plasticity in the congenitally blind. International Congress Series 1270:79-84

Lass NJ, Kotghek CL, Deem JF (1972) Oral two-point discrimination: further evidence of asymmetry on right and left side of selected oral structures. Percept Mot Skills 35:59-67

Maeyama T, Plattig KH (1989) Minimal two-point discrimination in human tongue and palate. Am J Otolaryngol 10:42-44

Marlow CD, Winkelmann RK, Gibilisco JA (1965) General sensory innervation of the human tongue. Anat Rec 152:503-512

Ptito M, Moesgaard SM, Gjedde A, Kupers R (2005) Cross-modal plasticity revealed by electrotactile stimulation of the tongue in the congenital blind. Brain 128:606-614

Ringel RL, Ewanowski SJ (1965) Oral perception: 1. two-point discrimination. J Speech Hear Res 8:389-397

Robineau F (2009) Etude d'un dispositif de guidage de geste chirurgical de ponction par stimulation lectrotactile linguale. PhD thesis, Université Joseph Fourier, Grenoble

Robineau F, Boy F, Orliaguet JP, Demongeot J, Payan Y (2007) Guiding the surgical gesture using an electro-tactile stimulus array on the tongue: A feasibility study. In: IEEE (ed) IEEE Transactions on Biomedical Engineering, 2007, vol 54, pp 711-717

Rodieck RW (2003) La Vision. Neurosciences \& Cognition, De Boeck

Sampaio E, Maris S, Bach-y-Rita P (2001) Brain plasticity: 'visual' acuity of blind persons via the tongue. Brain Res 908:204-207

Tatur G, Marc I, Lafon D, Dupeyron G, Bardin F, Dumas M (2011) Une approche fonctionnelle en vision prothetique : Etude préliminaire dans le contexte de la mobilité. In: Proceedings of the ASSISTH'11

Troxler IPV (1804) über das verschwinden gegebener gegenstände innerhalb unseres gesichtskreises. Ophthalmologische Bibliothek 2:1-53

Vuillerme N, Cuisinier R (2008) Head position-based electrotactile tongue biofeedback affects postural responses to achilles tendon vibration in humans. Exp Brain Res 186:503-508

Vuillerme N, Chenu O, Pinsault N, Moreau-Gaudry A, Fleury A, Demongeot J, Payan Y (2007) Pressure sensor-based tongue-placed electrotactile biofeedback for balance improvement-biomedical application to prevent pressure sores formation and falls. In: IEEE (ed) Conf. Proc. IEEE Eng. Med. Biol. Soc., 2007, vol 2007, pp 6114-6117

Vuillerme N, Chenu O, Pinsault N, Fleury A, Demongeot J, Payan Y (2008a) Can a plantar pressure-based tongue-placed electrotactile biofeedback improve postural control under altered vestibular and neck proprioceptive conditions ? Neuroscience 155:291-296

Vuillerme N, Pinsault N, Fleury A, Chenu O, Demongeot J, Payan Y, Pavan P (2008b) Effectiveness of an electro-tactile vestibular substitution system in improving upright postural control in unilateral vestibular-defective pa- 
tients. Gait \& Posture 28:711-715 


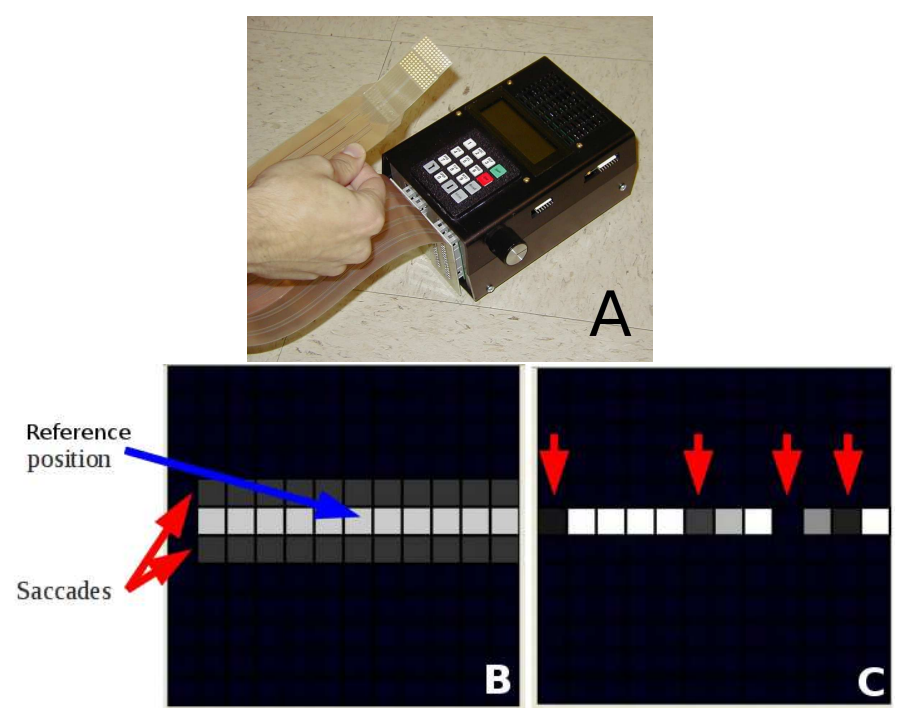

Fig. 1 Electrodes and their display. (A) $12 \times 12$ Electrode matrix and the control case of the TDU. Electrodes as displayed on the VDU with (B) a saccade effect and (C) a sparkle effect. In both cases, the reference line is a horizontal line of intensity $0.8(80 \%)$ displayed in the center of the electrode matrix. The line displayed in (B) appears mainly in the reference position where it is more intense, but also appears less intense on both sides of this position. The picture shown in $\mathrm{B}$ is an integration of 3 pictures taken at 3 different times because at each time only one line is displayed. In this example, taxels are lighted up until a distance of one taxel only due to limited saccades (e.g. 30\%), but under stronger saccades (e.g. 100\%) line drawings at larger distances are not uncommon. The picture shown in $(\mathrm{C})$, taken at a given time, shows that a line drawn with a sparkle effect is displayed as a series of points of varying intensities around an average intensity of $80 \%$. 


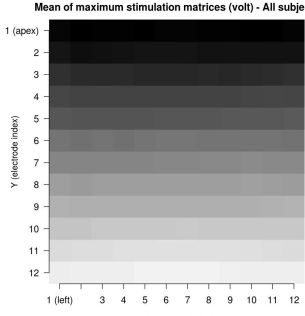

A

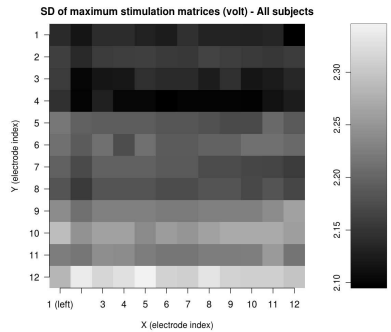

C

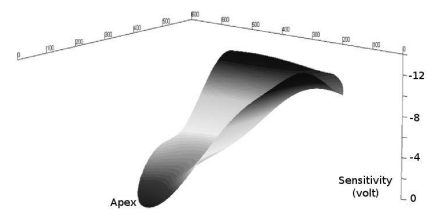

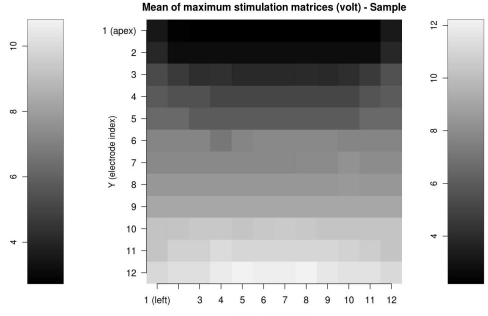
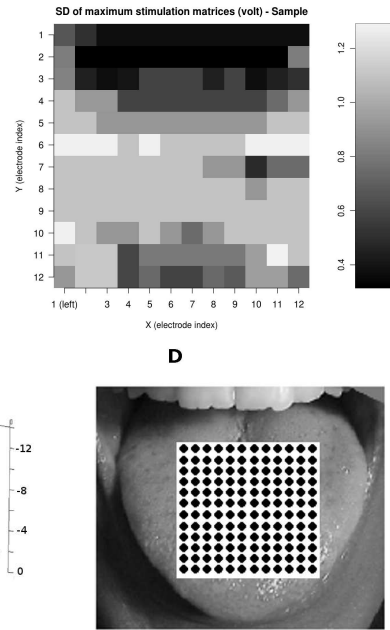

F

Fig. 2 Calibration and sensitivity maps. Average calibration matrices (in volts) of all subjects (A) and of one subject (B), and their respective standard deviations matrices (C) and (D). In (E), the sensitivity map of the tongue determined by Robineau (2009). In (F), a scheme of a $12 \times 12$ electrode matrix on the tongue of a subject. By comparing (A), (B) and $(\mathrm{E})$ one can recognize a similar antero-posterior increasing gradient of sensitivity on both calibration matrices and the sensitivity map, particularly in the example (B) where a secondary centro-lateral gradient is observed. The standard deviation matrices show that the subjects mostly correct voltages at the back of the tongue. 

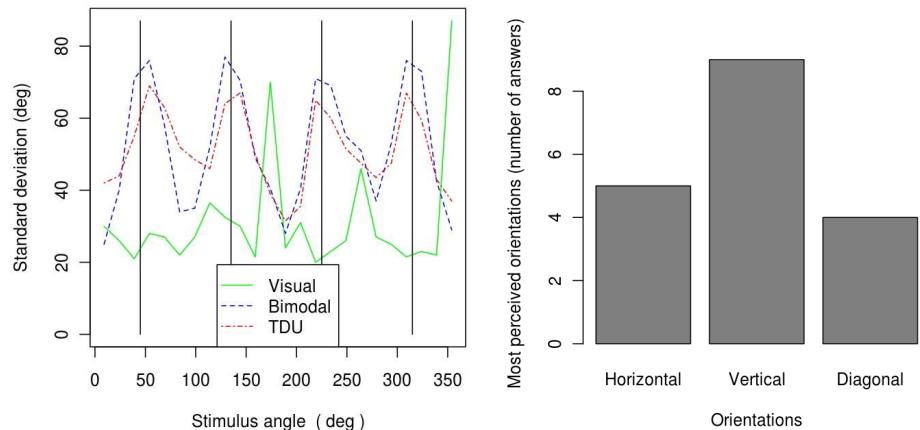

Fig. 3 Perception of directions. (Left) Angle standard deviations vs drawn angles. The hull of deviations of the drawings of 14 subjects is displayed as a function of the stimulus angle in the 3 modalities (VDU, TDU or both). These 3 curves were calculated by using a median filter with breaks of $15^{\circ}$ on the measured angle standard deviations up to $20^{\circ}$. Angle standard deviations increase when the stimulus angles get close to diagonals $\left(45^{\circ}, 135^{\circ}, 225^{\circ}\right.$ and $315^{\circ}$ ) in TDU and bimodal vision. (Right) Quiz on the perception of directions. We asked the subjects which directions the perceived at the best when using the TDU. Several answers were possible for each subject. Vertical lines appear clearly as the most perceived on the contrary to diagonals. 

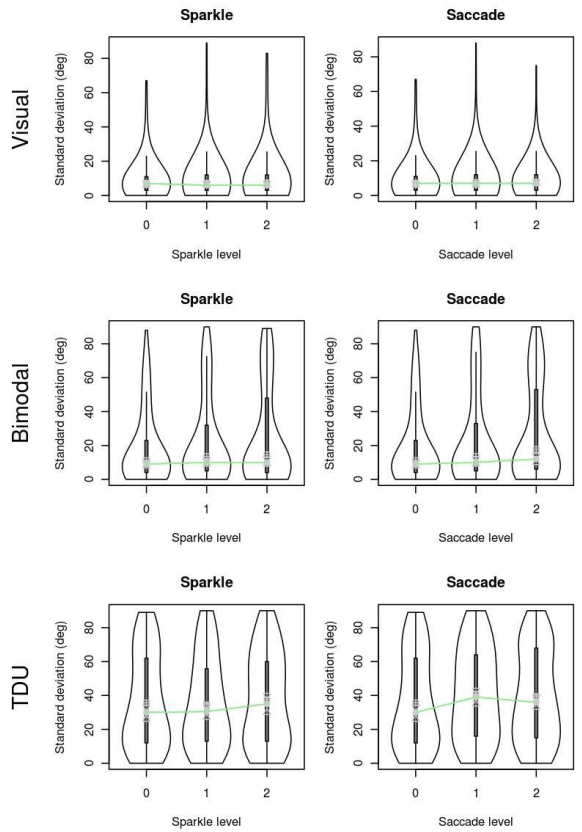

Fig. 4 Distributions of angle standard deviations. These violin plots allow to show the distribution of standard deviations of angles as a function of sparkle, saccades and mode of perception and the effect of these factors on their medians (more representative than means). One can observe notably the increase of standard deviations in bimodal and TDU vision, and the negative effect of saccades. The decrease of angle standard deviations due to sparkle is not easy to observe on this graphic and must be deduced from the analysis of variance given in Table 1. 

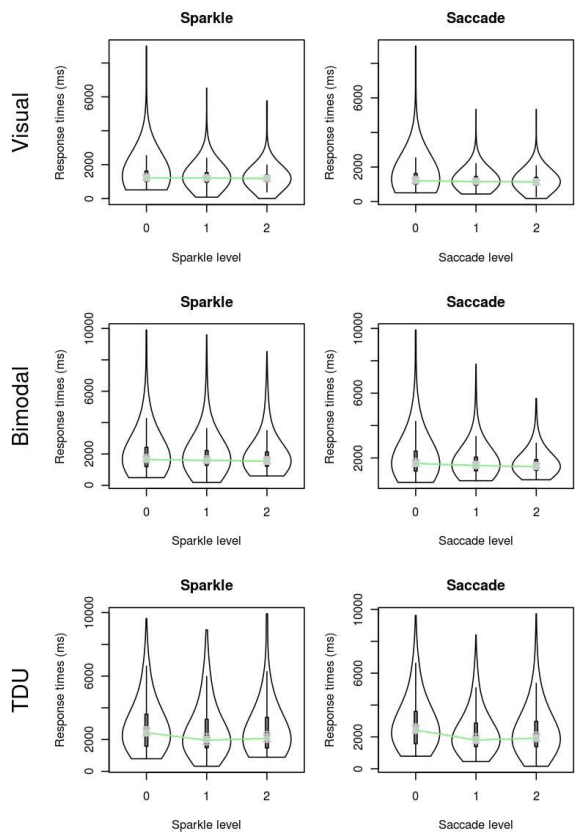

Fig. 5 Distributions (violin plots) of response times Similarly to Fig. 4 the graphics show the effect of sparkle, saccades and the mode of perception on the response times. At a first sight, response times do not vary drastically depending on the mode of vision (even if the analysis of variance finds a very significant effect). One can also see that saccades particularly have an effect in decreasing the response times. 
Table 1 Analysis of variance of angle standard deviations and response times. The table gives the F-statistics and p-values of several analyses of variance associated to different models (dependent variables are AnglesSD (standard deviations) and ResponseTimes ; factors are levels of Sparkle and Saccades (absence, moderate, strong), and the mode of perception Visu (VDU, bimodal, TDU)) and their a posteriori unilateral comparison tests based on pairwise Wilcoxon rank tests with Bonferroni p-value adjustment method (alternative hypotheses are indicated).

\begin{tabular}{|c|c|c|c|c|}
\hline $\begin{array}{l}\text { Models and Factors } \\
\text { (and alternative hypotheses) }\end{array}$ & \multicolumn{3}{|c|}{$A N O V A$} & $\begin{array}{c}\text { Test } \\
\text { p-value }\end{array}$ \\
\hline $\begin{array}{l}\text { AngleSD } \sim \text { Sparkle*Visu } \\
\text { Sparkle } \\
\text { Visu } \\
\text { Sparkle: Visu }\end{array}$ & $\begin{array}{l}\mathrm{F}(2,6921)= \\
\mathrm{F}(2,6921)= \\
\mathrm{F}(4,6921)=\end{array}$ & $\begin{array}{l}3.64 \\
929 \\
2.37\end{array}$ & $\begin{array}{l}2.6210^{-2} \\
\simeq 0 \\
4.9910^{-2}\end{array}$ & \\
\hline $\begin{array}{l}\text { AngleSD } \sim \text { Sparkle (TDU only) } \\
H_{1}: \text { Angles } S D_{\text {moderate }}<\text { Angles } S D_{\text {nosparkle }} \\
H_{1}: \text { Angles } S D_{\text {strong }}<\text { Angles } S D_{\text {nosparkle }} \\
\end{array}$ & $\mathrm{F}(2,2326)=$ & 4.65 & $9.610^{-3}$ & $\begin{array}{r}4.710^{-3} \\
23.010^{-2} \\
\end{array}$ \\
\hline $\begin{array}{l}\text { AnglesSD } \sim \text { Saccades*Visu } \\
\text { Saccades } \\
\text { Visu } \\
\text { Saccades: } \text { Visu }\end{array}$ & $\begin{array}{l}\mathrm{F}(2,6921)= \\
\mathrm{F}(2,6921)= \\
\mathrm{F}(4,6921)=\end{array}$ & $\begin{array}{l}9.3 \\
930 \\
4.53\end{array}$ & $\begin{array}{l}9.510^{-5} \\
\simeq 0 \\
1.1710^{-3}\end{array}$ & \\
\hline $\begin{array}{l}\text { AnglesSD } \sim \text { Saccades (TDU only) } \\
H_{1}: \text { Angles } S D_{\text {moderate }}>\text { Angles } S D_{\text {nosaccades }} \\
H_{1}: \text { Angles } S D_{\text {strong }}>\text { Angles } S D_{\text {nosaccades }}\end{array}$ & $\mathrm{F}(2,2326)=$ & 7.3 & $7.210^{-4}$ & $\begin{array}{l}3.010^{-4} \\
3.410^{-2}\end{array}$ \\
\hline $\begin{array}{l}\text { ResponseTime } \sim \text { Sparkle*Visu } \\
\text { Sparkle } \\
\text { Visu } \\
\text { Sparkle: } \text { Visu }\end{array}$ & $\begin{array}{l}\mathrm{F}(2,6921)= \\
\mathrm{F}(2,6921)= \\
\mathrm{F}(4,6921)=\end{array}$ & $\begin{array}{l}4.59 \\
673 \\
1.6\end{array}$ & $\begin{array}{l}1.0210^{-2} \\
\simeq 0 \\
17.210^{-2}\end{array}$ & \\
\hline $\begin{array}{l}\text { ResponseTime } \sim \text { Saccades*Visu } \\
\text { Saccades } \\
\text { Visu } \\
\text { Saccades: Visu }\end{array}$ & $\begin{array}{l}\mathrm{F}(2,6921)= \\
\mathrm{F}(2,6921)= \\
\mathrm{F}(4,6921)=\end{array}$ & $\begin{array}{l}33.5 \\
682 \\
4.84\end{array}$ & $\begin{array}{l}\simeq 0 \\
\simeq 0 \\
6.710^{-4}\end{array}$ & \\
\hline
\end{tabular}

